\title{
Time-Resolved Nonlinear Coupling between Orthogonal Flexural Modes of a Pristine GaAs Nanowire
}

\author{
D. Cadeddu, ${ }^{\dagger}$ F. R. Braakman, ${ }^{*}{ }^{\dagger}$ G. Tütüncüoglu, ${ }^{\dagger}$ F. Matteini, ${ }^{\ddagger}$ D. Rüffer, ${ }^{\ddagger}$ A. Fontcuberta i Morral, ${ }^{\ddagger}$ \\ and M. Poggio
}

${ }^{\dagger}$ Department of Physics, University of Basel, Klingelbergstrasse 82, 4056 Basel, Switzerland

${ }^{\ddagger}$ Laboratoire des Matériaux Semiconducteurs, École Polytechnique Fédérale de Lausanne, 1015 Lausanne, Switzerland

Supporting Information

ABSTRACT: We demonstrate nonlinear coupling between two orthogonal flexural modes of single as-grown GaAs nanowires. The resonant frequency of one mode can be shifted over many line widths by mechanically driving the other mode. We present time-domain measurements of the mode coupling and characterize it further by pump-probe experiments. Measurements show that a geometric nonlinearity causes the frequency of one mode to depend directly on the square amplitude of the other mode. Nearly degenerate orthogonal modes in nanowires are particularly interesting given their potential use in vectorial force sensing.
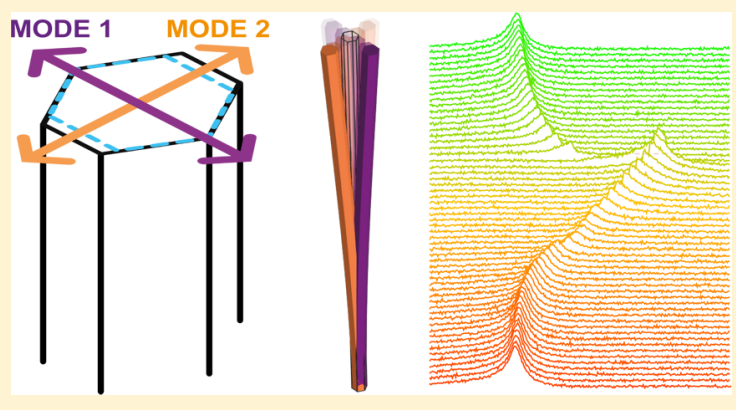

KEYWORDS: Nanowire, nonlinearity, mechanical mode coupling, nanomechanics

C oupling between different physical quantities lies at the very basis of the act of measurement. Coupling mechanisms in high-quality nanomechanical resonators are of particular interest since these are excellent candidates to study the transition of quantum to classical physics. ${ }^{1}$ Furthermore, the study of such coupling is essential for measurement techniques such as scanning probe microscopy as well as for the investigation of hybrid systems. Sufficiently strong coupling forms the basis for phenomena such as phonon-cavity physics, ${ }^{2}$ mechanically-induced transparency, ${ }^{2,3}$ and synchronization. ${ }^{4-6}$ It could also enable quantum nondemolition measurements of the displacement of one mode by measuring the frequency or phase of a coupled mode. ${ }^{7}$ Furthermore, the coupling of mechanical modes has various applications including in frequency and amplitude modulation, improving mechanical quality factors, ${ }^{9}$ in several parametric amplifications schemes, and in the implementation of mechanical logic. ${ }^{10,11}$ Mode coupling may also be used in the enhancement of mechanically detected mass, charge, and force sensitivity. ${ }^{12,13}$ For these reasons, such coupling has been studied in numerous top-down fabricated nanomechanical systems including single $e^{14,15}$ and double beam structures ${ }^{8,16}$ and membranes. ${ }^{17,18}$

Pristine bottom-up produced structures such as carbon nanotubes and nanowires showing great promise as ultrasensitive force transducers for scanning probe microscopy. ${ }^{19-22}$ Attractive features of these structures are their small dimensions, clean surfaces, the possibility of defect-free growth, and their relatively high mechanical resonance frequencies. Recent experiments demonstrated the use of nanowire force transducers in nanometer-scale magnetic resonance imaging ${ }^{20}$ and as ultrasensitive vectorial radiation force sensors. ${ }^{13}$
Moreover, nanowires can be grown as heterostructures, which makes them a very practical monolithic platform for studies of nanoscale hybrid systems. For example, the optical transitions of integrated self-assembled quantum dots in nanowires have recently been demonstrated to couple to the motion of their host nanowire through strain. ${ }^{23}$ For such experiments, measurements on two perpendicular flexural modes would add the unique possibility of obtaining vectorial information on force and strain fields, while mode coupling would allow to combine this with quantum nondemolition read-out. So far, mechanical mode coupling has been largely unobserved in bottom-up produced nanostructures. In one example, grown carbon nanotubes have been observed to exhibit coupling between flexural modes of different orders. ${ }^{24}$ However, these modes are neither perpendicular to each other nor are such nanotubes usable as singly clamped cantilever force transducers. In grown nanowires, orthogonal modes have been observed, ${ }^{13,19}$ but coupling has not yet been demonstrated or exploited.

In this Letter, we investigate nonlinear coupling of orthogonal flexural modes of as-grown GaAs nanowires. An asymmetry in the cross-section of a nanowire leads to the formation of two nondegenerate orthogonal modes. By driving one mode in its nonlinear regime of motion, the other mode is observed to shift in frequency. In a ringdown-type experiment, this leads to a distinct beating pattern in the time domain, where the beating period decreases with time. We show that the

Received: September 21, 2015

Revised: January 6, 2016

Published: January 19, 2016 
mode coupling and nonlinearity can be exploited for the implementation of frequency modulation, amplitude to frequency conversion, and logical operations.

The nanowires are grown perpendicularly on a (111)B GaAs substrate coated with $4 \mathrm{~nm} \mathrm{SiO}$ and are kept attached to this substrate (see Figure $1 \mathrm{~b}$ ). The growth proceeds by the catalyst-

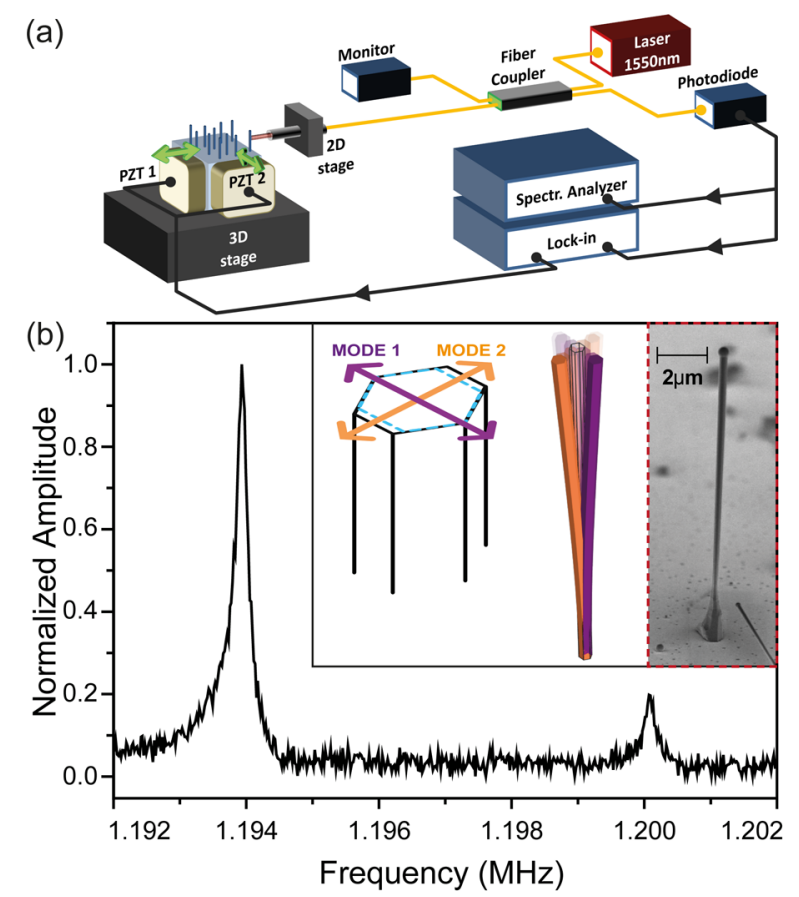

Figure 1. (a) Schematic of the measurement setup. (b) Frequency sweep at low driving amplitude showing the two mode resonances. The difference in the amplitude of the modes is related to the detection direction. Inset: scheme of the direction of the two modes in a nanowire with a small asymmetry in the cross-section (for reference, the blue dashed line indicates a symmetric hexagon) and a scanning electron micrograph of a GaAs nanowire with similar dimensions as the one studied.

free Gallium-assisted method ${ }^{25}$ in a DCA P600 solid source molecular beam epitaxy setup under a rotation of $7 \mathrm{rpm}$, with a growth rate of $0.5 \AA / \mathrm{s}$, and a substrate temperature of $630^{\circ} \mathrm{C}$. The crystal structure of the resulting nanowires is mostly zincblende, with a hexagonal cross-section. The produced nanowires have lengths up to $25 \mu \mathrm{m}$ and diameters of roughly 100 $\mathrm{nm}$. Keeping the nanowires pristine is important, as further processing can significantly degrade their structural and mechanical properties. Avoiding postprocessing of the nanowires minimizes the introduction of surface defects and ensures the rigid clamping of the nanowires to their substrate. Indeed, mechanical dissipation rates in grown nanowires have been reported to be much lower than for similar cantilevers produced in a top-down manner. ${ }^{19}$

The measurement setup is illustrated in Figure 1, panel a. The displacement of the nanowire is measured via a fiber-based method, ${ }^{19}$ in which the nanowire forms one reflecting interface of a low-finesse Fabry-Pérot interferometer, while the surface of a cleaved single-mode fiber forms the other interface. The sample is mounted on a stack of positioning stages for threeaxis translation control, which allows the nanowire to be placed in the focal point of an objective placed in front of the singlemode fiber. A fiber coupler is used to inject $1550 \mathrm{~nm}$ of light from a laser into the interferometer, which results in a maximum power incident on the nanowire of $\sim 5 \mu \mathrm{W}$. The large bandgap of GaAs compared to the energy of our laser allows us to avoid spurious heating of the nanowire through absorption. The light reflected by the interferometer is collected by a photodiode with a bandwidth of $5 \mathrm{MHz}$. Two perpendicularly placed, voltage-controlled piezoelectric transducers (PZTs) attached to the sample holder are used to drive oscillatory motion of the nanowires in two orthogonal directions. The two PZTs are independently actuated by two oscillators of a lock-in amplifier, and the same lock-in amplifier demodulates the response of the photodiode. The system is placed in a vacuum with pressure $<10^{-6} \mathrm{mbar}$ at room temperature.

A small asymmetry can be present in the cross-section of a grown nanowire, as shown schematically in Figure 1, panel b. Such an asymmetry leads to the formation of two nondegenerate flexural modes along the indicated directions (purple and orange arrows). As shown in Figure 1, panel b, the two modes studied here have resonant frequencies at $f_{1}=$ $\omega_{1} / 2 \pi=1.194 \mathrm{MHz}$ and $f_{2}=\omega_{2} / 2 \pi=1.200 \mathrm{MHz}$, which are reproduced numerically for a wire with a similar geometry, requiring an asymmetry in the cross-section of only $0.5 \%$. From ringdown measurements, as discussed later, we determine quality factors of 4300 and 5500, respectively, for the two modes.

The flexural motion of small structures such as nanowires and carbon nanotubes enters the nonlinear Duffing regime already for modest driving amplitudes, ${ }^{24,26}$ and consequently also strong coupling between flexural modes can be easily reached, as discussed later. To describe the nonlinear motion of the nanowire, we consider it as an isotropic, inextensible, Euler-Bernoulli beam. The large deformation of cantilever beam structures can give rise to geometric nonlinearities. ${ }^{27}$ Following the approach used by Crespo da Silva and Glynn ${ }^{28,29}$ (see Supporting Information), one then arrives at the following equation describing the displacement $a$ of steady-state motion of one of the two perpendicular modes for a small driving force at frequency $\omega$, with resonance frequency in the linear regime $\omega_{1}$ and damping rate $\eta_{1}$ :

$$
\left(\omega_{1}^{2}-\omega^{2}+i \eta_{1} \omega+\alpha_{1} a^{2}+\alpha_{1} b^{2}\right) a=\varepsilon F_{1}
$$

A symmetric equation can be written for the displacement of the second mode $b$ with linear resonant frequency $\omega_{2}$ and damping rate $\eta_{2}$. Here, $\alpha_{1} \equiv$ $\left(\frac{d_{1}}{L}\right)^{2} \int_{0}^{1} \xi(x)\left(\xi^{\prime}(x)\left(\xi^{\prime}(x) \xi^{\prime \prime}(x)\right)^{\prime}\right)^{\prime} \mathrm{d} x$ is the dimensionless version of the coupling coefficient $\tilde{\alpha}$ as defined in the Supporting Information, $L$ is the length, and $d_{1}$ the diameter of the nanowire, respectively. The first flexural mode shape is denoted by $\xi(x)$, where $x$ is the spatial coordinate along the nanowire axis. $F_{1}$ represents a driving force as defined in the Supporting Information and $\varepsilon \equiv \int_{0}^{1} \xi(x) \mathrm{d} x$.

The expression between parentheses on the left-hand side of eq 1 forms a frequency-dependent response factor to the driving, where the last two terms are due to the geometrical nonlinearity and shift the resonance frequency of mode 1 . The $a^{2}$ term results in a frequency shift $\Delta f_{\mathrm{d}}$, which is a quadratic function of the displacement of the same mode 1 . This is the same frequency shift one observes for a single-mode Duffing oscillator. $^{26}$ The $b^{2}$ term describes the mode coupling and result in a frequency shift $\Delta f_{\mathrm{c}}$ of mode 1, which varies quadratically with the displacement of the other mode $2 .^{30} \mathrm{~A}$ 
(a)
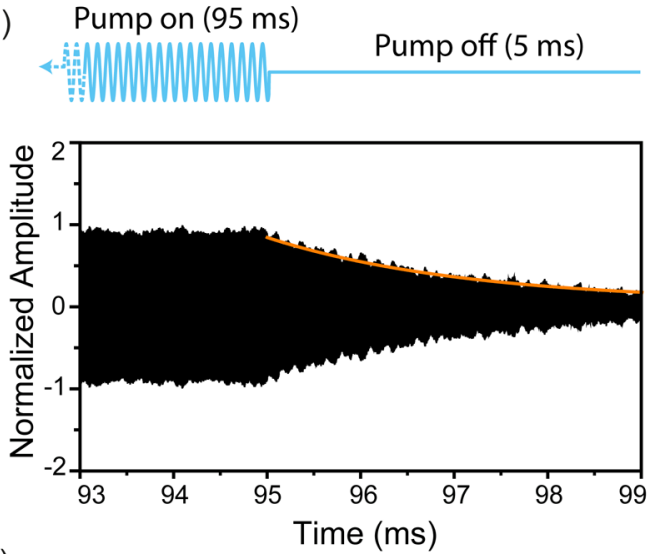

(c)

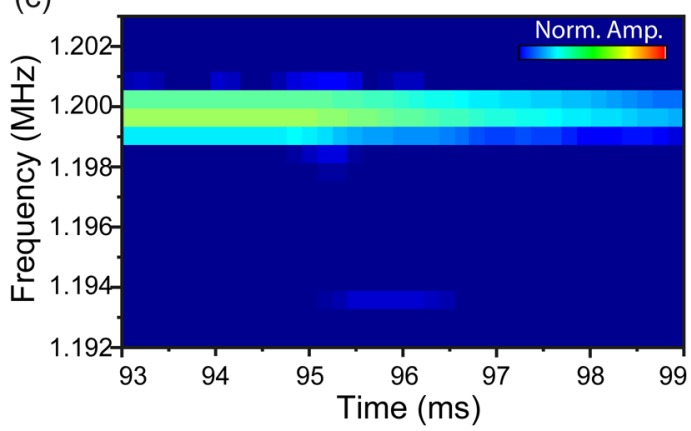

(b)
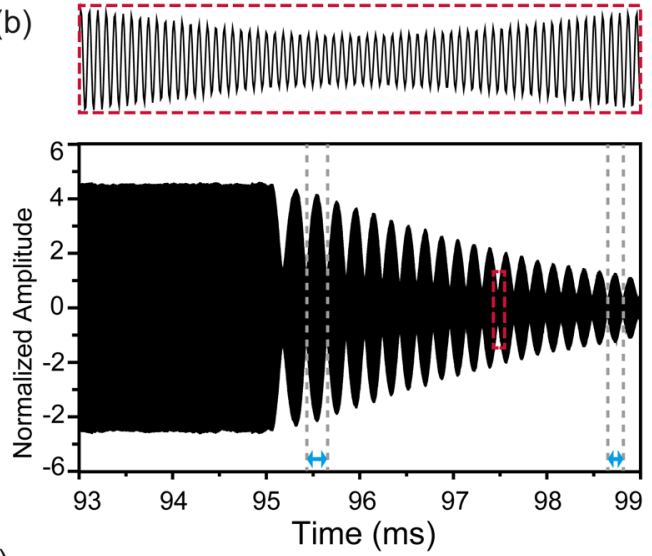

(d)

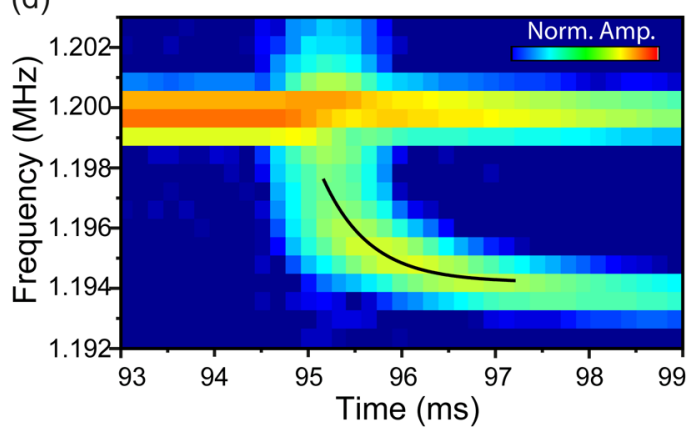

(e)

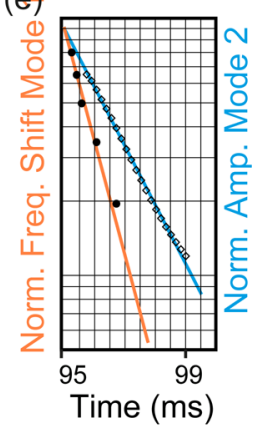

Figure 2. (a) Top: excitation scheme of the ringdown measurement. Bottom: ringdown with excitation at $f_{2}$ of $100 \mathrm{mV}$. An exponential fit to the decay is given by the orange curve. (b) Ringdown measurement with excitation at frequency $f_{2}$ and amplitude $2400 \mathrm{mV}$. Blue arrows highlight the varying beating period. The top panel shows a closeup of the displacement oscillations. (c, d) Short time Fourier transforms of the low and high amplitude ringdown measurements of panels a and $b$, respectively. In panel $d$, as the excitation is stopped, mode 2 is in its nonlinear regime as evidenced by the broadening of the peak and a slight shift toward higher frequencies. (e) Semilog plot of the normalized amplitude of mode 2 and the normalized frequency shift of mode 1 .

symmetric description applies to mode 2 . For this wire, we calculate $\tilde{\alpha}_{1}=6.4 \times 10^{4} \mathrm{~nm}^{-2} \mathrm{~s}^{-2}$. A similar value of coupling coefficient was found for other GaAs nanowires, ${ }^{26}$ and a slightly lower one can be found in literature for silicon nanowires. ${ }^{31}$ This value gives a coupling strength of $2.5 \mathrm{kHz}$ for an amplitude in mode 2 of just $10 \mathrm{~nm}$. By comparing this value with the 250 $\mathrm{Hz}$ line width of mode 1 , we can state that we are in a regime of strong coupling.

The frequency shift $\Delta f_{c}$ can be clearly observed in a ringdown experiment (see Figure 2a, top). In these measurements, a pump excitation is first applied at fixed amplitude and fixed frequency for $95 \mathrm{~ms}$ and then switched off, after which the decay of the excitation is observed for $5 \mathrm{~ms}$. The measurement is then repeated, and the results are averaged. Ringdown measurements with low driving amplitude show simple exponential decay and confirm the quality factors for both modes that were discussed before (Figure 2a). Next, we perform ringdown measurements at a higher driving amplitude at frequency $f_{2}$. Now a clear beating pattern is visible in the averaged time trace (see Figure $2 \mathrm{~b}$ ). The frequency of the beating is not constant in time but tends toward $f_{2}-f_{1}$ at the end of the decay. Qualitatively, this beating can be understood as follows: for high enough driving amplitudes, mode 1 is shifted enough in frequency through the coupling to overlap the pump frequency. Hence, this mode is also excited at the pump frequency. During the second part of the ringdown measurement, both modes decay at their own, time-dependent characteristic frequencies, resulting in beating. At the beginning of the decay, mode 2 oscillates at a high amplitude, causing mode 1 to be pulled to a higher frequency, that is, closer to $f_{2}$, thus reducing the beating frequency. As the amplitude of mode 2 decays, mode 1 moves back toward $f_{1}$, and the beating frequency therefore increases toward $f_{2}-f_{1}$.

In Figure 2, panels $\mathrm{c}$ and d, short-time Fourier transforms of the ringdown measurements are plotted. For low driving amplitude, only a frequency component at $f_{2}$ is present during both the excitation and decay parts of the measurement. For high driving amplitude, an additional frequency component develops at the time the excitation is stopped. This component is spectrally broad in the beginning, but over time narrows and shifts toward $f_{1}$, making it clear that it corresponds to an excitation of mode 1 . As described by eq $1, \Delta f_{c}$ is a quadratic function of the amplitude of mode 2, which decays exponentially with time constant $\tau_{2}=1 / \eta_{2}$. In Figure 2 , panel $\mathrm{e}$, the frequency shift of mode 1 , as well as the amplitude of mode 2 , is plotted as a function of time. Indeed, we find that $\Delta f_{\mathrm{c}}$ decays as $e^{-2 t / \tau_{2}}$, that is, twice as fast as the amplitude of mode 2 , confirming the nonlinear nature of the observed mode coupling.

When the pump excitation is applied on resonance with mode 1 , no beating pattern is observed, regardless of driving amplitude. This is consistent with the previous explanation since in this case the pump drive pushes mode 2 away from $f_{1}$, leading to no overlap of that mode with the excitation.

To investigate the dependence of $\Delta f_{c}$ on the displacement of the pumped mode in more detail, we perform pump-probe measurements. In the leftmost panel of Figure 3, the response of mode 1 is probed by sweeping with a weak excitation, while mode 2 is driven with a pump excitation, high enough to reach the nonlinear regime. Stepping the pump frequency changes 


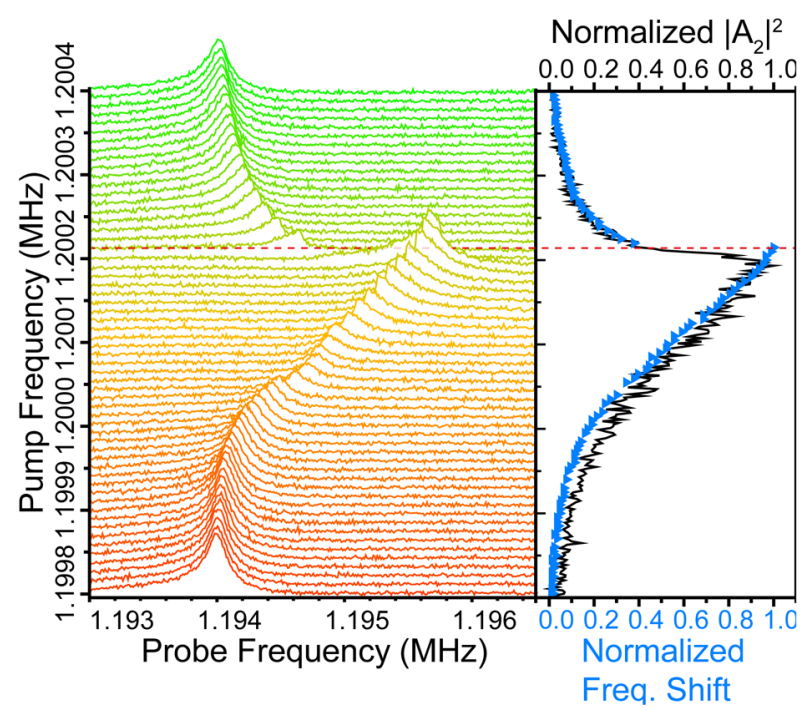

Figure 3. Left panel: frequency response of mode 1 for different pump frequencies around $f_{2}$, with driving amplitude $600 \mathrm{mV}$. Right panel: squared and normalized frequency response of mode 2 , at driving amplitude $600 \mathrm{mV}$ (black curve). Plotted on top are frequency shifts for maximum displacements of the mode 1 extracted from the left panel (blue triangles).

the displacement of the pumped mode according to its frequency response function and, through the mode coupling, maps this response function onto the frequency shift of the other mode. In the right panel of Figure 3, the squared and normalized frequency response of mode 2 is plotted (black curve) for the same driving amplitude as used in the left panel. Plotted on top of this (blue triangles) are the frequency shifts of the maximum response of mode 1, as shown in the left panel. The agreement between the curves confirms the quadratic dependence of the frequency pulling effect.

The shape of the resonance of the probed mode changes as the pump frequency is stepped. This provides a way to tune the effective nonlinearity of one mode by precisely controlling the motion in the other, orthogonal mode. By using perturbation theory, one can write the effective nonlinearity constant of a mode in terms of the derivative of the squared displacement in the other mode with respect to the driving frequency:

$$
\alpha_{1, \mathrm{eff}}=\alpha_{1}\left(1-\alpha_{1} \frac{\partial|b|^{2}}{\partial \omega}\right)
$$

Mode 2 shows a jump in its response around the frequency indicated in Figure 3 by the red dashed line. Because of this effect, the last term in eq 2 changes sign at this frequency, which results in a negative effective nonlinearity (sharkfin-shape with jump on lower flank) of the probed mode for lower pump frequencies and a positive effective nonlinearity (sharkfin-shape with jump on higher flank) for higher pump frequencies.

Similarly, the amplitude response function of the pumped mode can be mapped onto the frequency shift of the other mode by stepping the pump amplitude at fixed frequency. Figure 4, panel a displays the frequency response functions of mode 2 in the linear and nonlinear regime. Fixing the pump frequency at $f_{2}$ and varying the pump amplitude result in frequency shifts of mode 1 as shown in Figure 4, panel b. In Figure 4, panel c, the pump frequency is set to a value higher than $f_{2}$. Varying the pump amplitude initially results in a small, quadratic frequency shift of mode 1 . As the pump amplitude is increased, mode 2 enters its nonlinear regime and becomes bistable, showing a jump in its response at a frequency that increases with increasing pump amplitude. For high enough amplitude, this frequency reaches the pump frequency, allowing mode 2 to jump to its high-amplitude branch. This jump is observed as a corresponding jump in frequency shift of mode 1.

For relatively low displacements of mode 2 , the frequency shift in both cases shows a quadratic dependence (see insets of Figure $4 b, c)$. The deviations from this behavior at higher pump amplitudes are most likely related to optical compression in the interferometric detector, as well as mechanical mixing due to the presence of both pump and probe drives, and possibly due to the wire entering a strong bending regime of motion. ${ }^{32}$

We confirm the orthogonal nature of the two modes by using two perpendicularly mounted PZTs to drive the pump excitation (as shown in Figure 1b). From the magnitude of the frequency shifts (solid and dashed data sets in Figure $4 b$, for PZT2 and PZT1, respectively), we infer that PZT2 drives mode
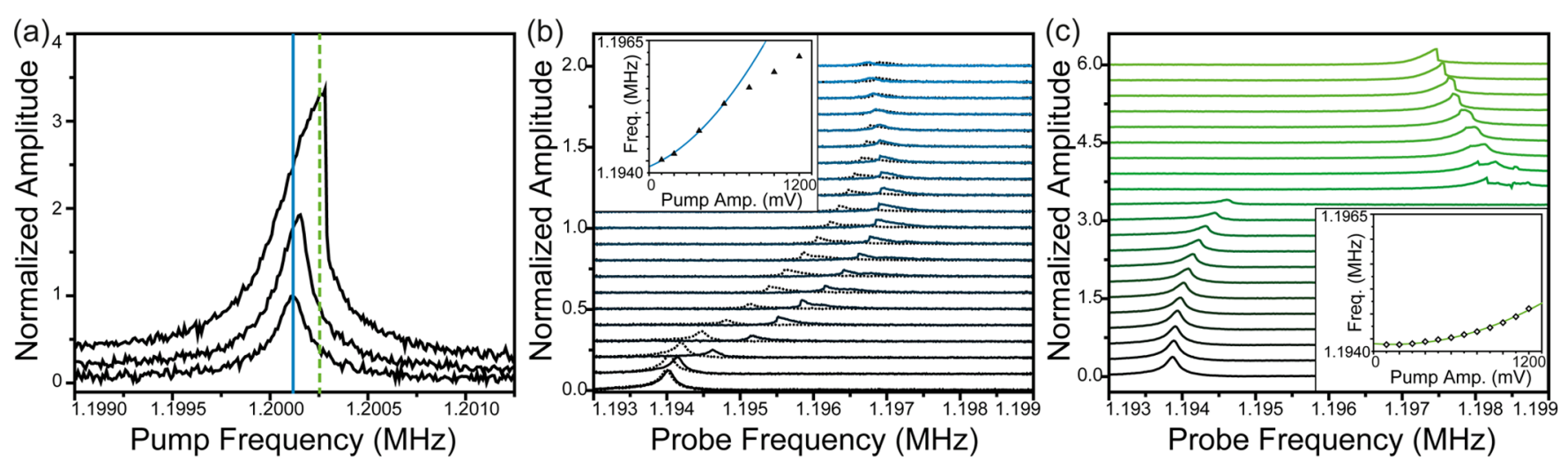

Figure 4. (a) Pump-probe measurement scheme with the pump drive at two different fixed frequencies: at the resonance frequency of mode 2 in the linear regime (solid blue line) and at a frequency higher than the critical frequency of mode 2 in the nonlinear regime (dashed green line). (b) Frequency response sweeps of the probed mode with the pump amplitude increasing from bottom to top. The solid and dashed black lines correpond to pumping with PZT2 and PZT1 (see Figure 1a), respectively. Inset: frequency of maximum response of probed mode (from dashed curves), plotted as a function of pump amplitude, for relatively low pump amplitudes. The curve is a fit to a quadratic function. (c) Frequency sweeps of the probed mode with the pump drive frequency as indicated by the dashed green line in panel a. Inset: frequency of maximum response of probed mode, plotted as a function of pump amplitude, for relatively low pump amplitudes. The curve is again a fit to a quadratic function. 
2 more than does PZT1. Similarly, by probing the response of mode 1 with each PZT, we determine that PZT1 drives mode 1 harder than does PZT2.

The ability to map the displacements and oscillation frequencies of one mode onto the frequency of another mode can be used to implement amplitude to frequency conversion, frequency modulation, and frequency tuning. This type of mode coupling and nonlinearity can also be used to perform mechanical logic. As a proof-of-principle, we demonstrate mechanical OR and NOR gates in the Supporting Information. The hysteresis due to the nonlinearity of the modes also provides a straightforward way to store these logical output states. $^{10,11}$

In conclusion, we have shown that two orthogonal flexural modes of a GaAs nanowire can couple through nonlinear terms in the motion. The mode coupling is clearly visible in ringdown measurements, where we observe a beating pattern with frequency equal to the difference in mode frequencies. We furthermore demonstrate that the frequency shift of one mode is proportional to the square amplitude of the coupled mode, which confirms the nonlinear nature of the coupling. This system allows for the implementation of mechanical logic and memory. Finally, a prospective use of the two orthogonal modes in the nanowires lies in bidimensional sensing. By entering the nonlinear regime of motion in each mode, a force sensitivity of $\sim 100 \mathrm{zN} / \sqrt{\mathrm{Hz}}^{26}$ could in principle be reached in both orthogonal directions simultaneously. Such a bidimensional sensor has been used before to measure the nonconservative nature of radiation pressure, ${ }^{13}$ but could also be used to detect vector force gradients on a sample surface, for example, in an atomic force microscopy setup. The mode coupling could enhance such measurements by allowing twodimensional information to be obtained through the read-out of a single mode.

\section{ASSOCIATED CONTENT}

\section{S Supporting Information}

The Supporting Information is available free of charge on the ACS Publications website at DOI: 10.1021/acs.nanolett.5b03822.

Equations of motions; mechanical logic (PDF)

\section{AUTHOR INFORMATION}

\section{Corresponding Author}

*E-mail: floris.braakman@unibas.ch.

\section{Author Contributions}

D.C., F.R.B., and M.P. conceived and designed the experiment. The nanowires were provided by G.T. D.R., F.M., and A.F. The measurements were performed by D.C. and F.R.B. D.C., F.R.B., and M.P. analyzed and interpreted the data. D.C., F.R.B., and M.P. wrote the manuscript. All authors discussed the results and contributed to the manuscript.

Notes

The authors declare no competing financial interest.

\section{ACKNOWLEDGMENTS}

We thank Sascha Martin for technical support. This work is supported by an ERC Grant (NWscan, Grant No. 334767), the Swiss Nanoscience Institute (Project No. P1207), the National Centre of Competence in Research, Quantum Science and Technology, and the Canton Aargau.

\section{REFERENCES}

(1) Zurek, W. Progress in Mathematical Physics 2007, 48, 1-31.

(2) Mahboob, I.; Nishiguchi, K.; Okamoto, H.; Yamaguchi, H. Nat. Phys. 2012, 8, 387-392.

(3) Weis, S.; Rivière, R.; Deléglise, S.; Gavartin, E.; Arcizet, O.; Schliesser, A.; Kippenberg, T. J. Science 2010, 330, 1520-1523.

(4) Shim, S. B.; Imboden, M.; Mohanty, P. Science 2007, 316, 95-99.

(5) Matheny, M. H.; Grau, M.; Villanueva, L. G.; Karabalin, R. B.; Cross, M. C.; Roukes, M. L. Phys. Rev. Lett. 2014, 112, 014101.

(6) Walter, S.; Nunnenkamp, A.; Bruder, C. Ann. Phys. (Berlin, Ger.) 2015, 527, 131.

(7) Santamore, D. H.; Doherty, A. C.; Cross, M. C. Phys. Rev. B: Condens. Matter Mater. Phys. 2004, 70, 144301.

(8) Karabalin, R. B.; Cross, M. C.; Roukes, M. L. Phys. Rev. B: Condens. Matter Mater. Phys. 2009, 79, 165309.

(9) Venstra, W. J.; Westra, H. J. R.; Van der Zant, H. S. J. Appl. Phys. Lett. 2011, 99, 151904.

(10) Mahboob, I.; Yamaguchi, H. Nat. Nanotechnol. 2008, 3, 275279.

(11) Yao, A.; Hikihara, T. Appl. Phys. Lett. 2014, 105, 123104.

(12) Gil-Santos, E.; Ramos, D.; Martínez, J.; Fernández-Regúlez, M.; García, R.; San Paulo, Á; Calleja, M.; Tamayo, J. Nat. Nanotechnol. 2010, 5, 641-645.

(13) Gloppe, A.; Verlot, P.; Dupont-Ferrier, E.; Siria, A.; Poncharal, P.; Bachelier, G.; Vincent, P.; Arcizet, O. Nat. Nanotechnol. 2014, 9, 920-926.

(14) Lulla, K. J.; Cousins, R. B.; Venkatesan, A.; Patton, M. J.; Armour, A. D.; Mellor, C. J.; Owers-Bradley, J. R. New J. Phys. 2012, 14, 113040.

(15) Truitt, P. A.; Hertzberg, J. B.; Altunkaya, E.; Schwab, K. C. J. Appl. Phys. 2013, 114, 114307.

(16) Faust, T.; Rieger, J.; Seitner, M. J.; Krenn, P.; Kotthaus, J. P.; Weig, E. M. Phys. Rev. Lett. 2012, 109, 037205.

(17) Flowers-Jacobs, N. E.; Hoch, S. W.; Sankey, J. C.; Kashkanova, A.; Jayich, A. M.; Deutsch, C.; Reichel, J.; Harris, J. G. E. Appl. Phys. Lett. 2012, 101, 221109.

(18) Antoni, T.; Makles, K.; Braive, R.; Briant, T.; Cohadon, P.; Sagnes, I.; Robert-Philip, I.; Heidmann, A. Europhys. Lett. 2012, 100, 68005 .

(19) Nichol, J. M.; Hemesath, E. R.; Lauhon, L. J.; Budakian, R. Appl. Phys. Lett. 2008, 93, 193110.

(20) Nichol, J. M.; Naibert, T. R.; Hemesath, E. R.; Lauhon, L. J.; Budakian, R. Phys. Rev. X 2013, 3, 031016.

(21) Feng, X. L.; He, R.; Yang, P.; Roukes, M. L. Nano Lett. 2007, 7, 1953-1959.

(22) Poggio, M. Nat. Nanotechnol. 2013, 8, 482-483.

(23) Montinaro, M.; Wüst, G.; Munsch, M.; Fontana, Y.; RussoAverchi, E.; Heiss, M.; Fontcuberta i Morral, A.; Warburton, R. J.; Poggio, M. Nano Lett. 2014, 14, 4454-4460.

(24) Eichler, A.; Del Álamo-Ruiz, M.; Plaza, J. A.; Bachtold, A. Phys. Rev. Lett. 2012, 109, 025503.

(25) Colombo, C.; Spirkoska, D.; Frimmer, M.; Abstreiter, G.; Fontcuberta i Morral, A. Phys. Rev. B: Condens. Matter Mater. Phys. 2008, 77, 155326.

(26) Braakman, F. R.; Cadeddu, D.; Tütüncüoglu, G.; Rüffer, D.; Matteini, F.; Fontcuberta i Morral, A.; Poggio, M. Appl. Phys. Lett. 2014, 105, 173111.

(27) Malatkar, P. Nonlinear Vibrations of Cantilever Beams and Plates. Ph.D. Dissertation, Virginia Polytechnic Institute and State University, Blacksburg, VA, 2003.

(28) Crespo da Silva, M. R. M.; Glynn, C. C. J. Struct. Mech. 1978, 6 (4), 437-448.

(29) Crespo da Silva, M. R. M.; Glynn, C. C. J. Struct. Mech. 1978, 6 (4), 449-461.

(30) Westra, H. J. R.; Poot, M.; Van der Zant, H. S. J.; Venstra, W. J. Phys. Rev. Lett. 2010, 105, 117205.

(31) Nichol, J. M.; Hemesath, E. R.; Lauhon, L. J.; Budakian, R. Appl. Phys. Lett. 2009, 95, 123116. 
(32) Sapmaz, S.; Blanter, Y. M.; Gurevich, L.; Van der Zant, H. S. J.

Phys. Rev. B: Condens. Matter Mater. Phys. 2003, 67, 235414. 